\title{
To See or Not to See: Prestimulus $\alpha$ Phase Predicts Visual Awareness
}

\author{
Kyle E. Mathewson, ${ }^{1,2}$ Gabriele Gratton, ${ }^{1,2}$ Monica Fabiani, ${ }^{1,2}$ Diane M. Beck, ${ }^{1,2}$ and Tony Ro ${ }^{3}$ \\ ${ }^{1}$ Department of Psychology and ${ }^{2}$ Beckman Institute, University of Illinois at Urbana-Champaign, Urbana, Illinois 61801, and ${ }^{3}$ Program in Cognitive \\ Neuroscience and Department of Psychology, The City College of the City University of New York, New York, New York 10031
}

We often fail to see something that at other times is readily detectable. Because the visual stimulus itself is unchanged, this variability in conscious awareness is likely related to changes in the brain. Here we show that the phase of EEG $\alpha$ rhythm measured over posterior brain regions can reliably predict both subsequent visual detection and stimulus-elicited cortical activation levels in a metacontrast masking paradigm. When a visual target presentation coincides with the trough of an $\alpha$ wave, cortical activation is suppressed as early as $100 \mathrm{~ms}$ after stimulus onset, and observers are less likely to detect the target. Thus, during one $\alpha$ cycle lasting 100 ms, the human brain goes through a rapid oscillation in excitability, which directly influences the probability that an environmental stimulus will reach conscious awareness. Moreover, ERPs to the appearance of a fixation cross before the target predict its detection, further suggesting that cortical excitability level may mediate target detection. A novel theory of cortical inhibition is proposed in which increased $\alpha$ power represents a "pulsed inhibition" of cortical activity that affects visual awareness.

\section{Introduction}

Given a constant set of stimulus parameters, visual perception can be highly variable, for reasons that are poorly understood. For instance, at a maximally effective masking stimulus onset asynchrony (SOA) of $50 \mathrm{~ms}$, a metacontrast backwards mask is not always effective in diminishing the visibility of a preceding target (Breitmeyer and Ogmen, 2000; Di Lollo et al., 2000). Presumably, this variability in visual perception is a consequence of different brain states and resultant responsiveness to the visual targets. Whereas much research has investigated the differential neural activity elicited by detected and undetected targets, and by masks of variable efficacy (Lau and Passingham, 2002; Haynes et al., 2005; van Aalderen-Smeets et al., 2006; Del Cul et al., 2007; Fahrenfort et al., 2007), few investigations have specifically examined the differential prestimulus electrophysiological activity that leads observers to detect or not detect targets on any given trial.

Several studies have shown that both top-down (Ramachandran and Cobb, 1995; Tata, 2002; Boyer and Ro, 2007) and stimulus-driven (Shelley-Tremblay and Mack, 1999) attention play an important role in the effectiveness of visual masking. Other studies have suggested the possible instantiation of attentional influences on visual processing by suppression of rhythmic oscillations in the electroencephalogram (EEG) $\alpha$ frequency range $(8-12 \mathrm{~Hz})$ (Worden et al., 2000; Fries et al., 2001; Sauseng et al., 2005; Thut et al., 2006; Romei et al., 2008). For example, Ergenoglu et al. (2004) found that decreases in $\alpha$ power before

\footnotetext{
Received Aug. 20, 2008; revised Dec. 26, 2008; accepted Jan. 15, 2009.

This work was supported by a Natural Science and Engineering Research Council of Canada Fellowship to K.E.M. and National Institute of Mental Health Grant MH080182 to G.G. We thank Kathy Low for technical assistance and Tanya Stanley and Jayme Jones for assistance in subject running.

Correspondence should be addressed to Tony Ro at the above address. E-mail: tro@ccny.cuny.edu. D0I:10.1523/JNEUROSCI.3963-08.2009

Copyright $\odot 2009$ Society for Neuroscience $\quad$ 0270-6474/09/292725-08\$15.00/0
}

target onset are associated with increases in visual target detection. Based on these results, we hypothesized that changes in $\alpha$ may be associated with the variability in detectability observed in metacontrast masking.

Lindsley (1952) proposed that the phase of $\alpha$ might represent oscillations in cortical excitability. This hypothesis is consistent with recent evidence showing relationships between EEG slowwave's phase and increases in cortical excitability (Lakatos et al., 2008), between $\alpha$ phase and increases in event-related potential (ERP) amplitude (Trimble and Potts, 1975; Jansen and Brandt, 1991; Haig and Gordon, 1998; Barry et al., 2003, 2004; Kruglikov and Schiff, 2003), and faster responses to phase-locked stimuli (Callaway and Yeager, 1960). Despite this evidence, no studies have directly examined the influences of the phase of $\alpha$ on awareness of a visual stimulus.

Here, we investigated the neural activity associated with increased target detectability in a backwards metacontrast masking paradigm. Using constant stimulus parameters, we examined both the ERPs elicited by a fixation cross signaling the beginning of each trial, as well as the power and phase of the $\alpha$ activity in the period after fixation and before the onset of the masked targets. Additionally, we assessed how the ERP activity elicited by the detected and undetected targets and masks relates to both these prestimulus differences in neural activity and to visual awareness.

\section{Materials and Methods}

Subjects. Eighteen subjects (13 females; age range 18-30) gave informed consent as approved by the Institutional Review Board at the University of Illinois at Urbana-Champaign. Six subjects were not included in the analysis because their target detection rate was $>90 \%$ or $<10 \%$ and thus did not have enough trials in one of the two critical conditions. A seventh subject was excluded because of an extremely high false alarm rate (85\%). Thus the behavioral and electrophysiological (EEG and ERP) data from 11 subjects ( 8 females; age range 18-29) were considered. 
Stimuli and procedures. Subjects were comfortably seated $57 \mathrm{~cm}$ away from and at eye level with a 20 -inch Sony Trinitron CRT monitor, with a refresh rate of $85 \mathrm{~Hz}$. Figure 1 shows the stimulus dimensions and a typical trial sequence presented to each subject using E-prime presentation software (Psychology Software Tools). Each trial began with a black fixation cross presented at the center of the monitor for $247 \mathrm{~ms}$. The fixation cross was followed by a blank screen lasting $400 \mathrm{~ms}$. The dark gray target disk was then centrally presented for $11.7 \mathrm{~ms}$ with a $47.0 \mathrm{~ms}$ interstimulus interval (ISI) before the dark gray annulus mask appeared for $23.4 \mathrm{~ms}$ (these timings represent multiples of $11.7 \mathrm{~ms}$, which is the duration of one frame at an $85 \mathrm{~Hz}$ refresh rate). All stimuli were presented on a light gray background. Subjects then had 1500 $\mathrm{ms}$ before the next trial began, during which a response was made to indicate whether or not they had seen the target. Responses were made on an E-prime button box (1, detected; 5, undetected) and subjects received no feedback regarding their accuracy. On average, subjects failed to respond on $1 \%$ of trials, which were excluded from further analysis.

Each subject completed 16 blocks, each consisting of 72 trials. Short rest breaks, the duration of which was controlled by the subjects, were given between blocks. Trial types were randomly intermixed with the restriction that half of the trials (576) contained both the target and mask, whereas on one quarter of the trials (288) the masks appeared with a blank screen in place of the target and on the remaining quarter ( 288 trials) the target appeared with a blank screen in place of the mask. Subjects were given both visual and verbal instructions and performed a practice block consisting of 40 trials before the 16 experimental blocks began.

EEG and ERP recording and analysis. The EEG was recorded from 21 $\mathrm{Ag} / \mathrm{AgCl}$ electrodes using an electrode cap with a standard 10/20 system layout. Electrodes were referenced to the left mastoid online, and were re-referenced to an arithmetically derived average mastoid reference offline. Electrodes above and below the left eye, and on the outer canthi of each eye, measured the subject's bipolar vertical and horizontal EOG activity. Electrode impedance was kept at $<10 \mathrm{k} \Omega$.

The EEG was first filtered online with a $0.01-30 \mathrm{~Hz}$ bandpass and sampled at $200 \mathrm{~Hz}$. Off line, the EEG was again low-pass filtered at $25 \mathrm{~Hz}$. The data were then segmented into $2223 \mathrm{~ms}$ epochs, time-locked to the onset of the initial fixation for each trial. After removing trials with A/D saturation, ocular artifacts were detected and corrected (Gratton et al., 1983 ) and trials with artifacts $>250 \mu \mathrm{V}$ were discarded. In total, $<4 \%$ of the trials were discarded because of artifacts. The proportion of recording time points classified as eye blinks in the $650 \mathrm{~ms}$ period before the onset of the target did not differ between undetected $(M=0.0022)$ and detected targets $\left(M=0.0023, t_{(10)}=0.21\right.$, n.s. $)$.

Separate grand average waveforms were computed for detected and undetected target trials. The waveforms were baseline corrected using the activity during the $200 \mathrm{~ms}$ before fixation onset to derive fixation ERPs. Time windows for the P1 (90-160 ms), N1 (150-230 ms), and P2 (200$250 \mathrm{~ms}$ ) components were chosen to be centered around visually identified component peaks in the grand average waveforms across conditions. For each component the mean voltage within the corresponding time window was computed for each subject, separately for detected and undetected trials.

To remove the activity elicited by the mask without removing activity resulting from the interaction of mask and target, the mask-only average was subtracted from each target-plus-mask average (see Del Cul et al., 2007 for a justification of this procedure). These waveforms were then time-locked to the onset of the target and baseline-corrected to the 200 $\mathrm{ms}$ preceding its onset. For this analysis, the same time windows men-
A

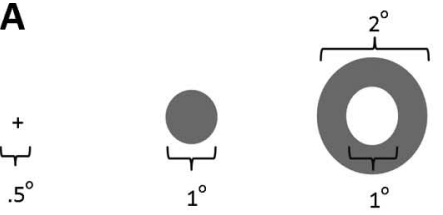

$1^{\circ}$

Figure 1. $\quad \boldsymbol{A}$, Spatial dimensions of the stimuli, which were presented to subjects at the center of the screen. $\boldsymbol{B}$, Individual tria timeline with durations of each screen presentation. Note that on $25 \%$ of the trials the target was replaced with a blank screen to

tioned above for the fixation were used for the target P1, N1, P2. An additional time window (300-500 ms) was chosen to analyze the P3. For each of these time windows the mean voltages for detected and undetected targets were then computed. For both fixation and target waveforms, the mean voltages for each time window for detected and undetected targets were compared using paired one-tailed $t$ tests because previous studies (Del Cul et al., 2007; Pourtois et al., 2006) have shown larger ERP components for detected compared with undetected stimuli (i.e., $\mathrm{H}_{0}$ : $\left.\mu \mathrm{V}_{\text {Detected }} \leq \mu \mathrm{V}_{\text {Undetected }}\right)$. The test of each component was performed at a single electrode site chosen based on extant literature (e.g., P3 was tested at $\mathrm{Pz}, \mathrm{N} 1$ at $\mathrm{Fz}$ ).

$\alpha$ Power and phase analysis. A discrete Fourier Transform with no taper was applied to the raw EEG data from the $200 \mathrm{~ms}$ period preceding target onset for the detected and undetected trials at electrode Pz. This time window was chosen because initial analyses suggested that the fixation offset affected EEG activity for $\sim 200 \mathrm{~ms}$. This electrode was chosen based on where $\alpha$ effects have been maximal in previous studies (Ergenoglu et al., 2004). Both the power and the phase (in degrees) at a frequency of 10 $\mathrm{Hz}$ were extracted from the transformed data for each trial, condition, and subject. With this interval duration only frequencies that are multiples of $5 \mathrm{~Hz}$ can be measured; hence the choice of $10 \mathrm{~Hz}$, which could not be varied across individuals. For each subject, all trials were then divided into quartile bins of log-transformed power at electrode Pz. The detection rate in each of these bins was computed separately for each subject. A test of linear trend in detection across bins was run using a repeatedmeasure ANOVA.

For an additional test of the robustness of this finding, the average $\alpha$ power for detected targets was directly compared with that for undetected targets using a two-tailed $t$ test. To test for changes in $\alpha$ power over the course of a block, the average log-transformed $\alpha$ power for the $200 \mathrm{~ms}$ period before target onset was separately computed for all trials in the first half and second half of each block. These measures were obtained for each subject, collapsed across conditions, and submitted to a paired twotailed $t$ test at the Pz electrode.

For analysis of the phase of $\alpha$, trials were sorted based on the phase of $\alpha$ activity at electrode $\mathrm{Pz}$ at target onset into two opposite bins $\left(225-45^{\circ}\right.$ and $45-225^{\circ}$ ). These bins were chosen to be orthogonal to the mean phases for undetected $\left(134.6^{\circ}\right)$ and detected targets $\left(345.9^{\circ}\right)$ for the high 
$\alpha$ power trials. For each of these bins, the average detection rate was calculated for each subject. Because any effect of phase should only be present in trials with sufficient $\alpha$ power, trials were further divided into high- and low-power bins using a median split. The main effects of power and phase, as well as their interaction, were then tested using a two-way repeated-measures ANOVA.

An additional analysis of the phase data was run using the Cartesian coordinates in the complex space in a multivariate approach. This approach was chosen because it combines analysis of phase and amplitude: this is crucial to prevent trials with small $\alpha$ amplitude (and therefore substantially random phase) from exerting an undue influence on the results. Furthermore, only when $\alpha$ reaches sufficient amplitude can its phase be expected to be of any significance. Practically, for each trial we evaluated the complex values derived from the Fourier transform at 10 $\mathrm{Hz}$ using Cartesian coordinates rather than the polar coordinates (distance from origin, or amplitude, and angle, or phase). Differences between the average coordinates for detected and undetected trials were then computed for each subject. The resultant $\mathrm{X}$ and $\mathrm{Y}$ components were then submitted to a Hotelling's bivariate $F$ test for a difference from zero, with a significant difference from zero indicating that the bivariate distributions differed between detected and undetected trials.

All processing and analyses were performed using custom scripts written in Matlab (The MathWorks) and with the EEGLAB toolbox (Delorme and Makeig, 2004). Apart from the detected vs undetected ERP comparisons, in which we indicate the use of one-tailed statistical comparisons based on previous findings (Pourtois et al., 2006; Del Cul et al., 2007), all other statistics were conducted using two-tailed tests.

\section{Results}

\section{Behavior}

The 11 subjects had an average detection rate of $70 \%$ on the target-plus-mask trials ( $\mathrm{SD}=14 \%$; range $=46-82 \%$ ). On the mask-only trials, the average false alarm rate was $14 \%(\mathrm{SD}=7 \%$; range $=3-24 \%)$. Signal detection analyses yielded an average criterion of $0.51(\mathrm{SD}=0.63$; range $=-0.36$ to 1.64$)$ and an average $d^{\prime}$ of $1.74(\mathrm{SD}=0.41$; range $=1.47-2.82)$, indicating that all subjects, including those with high false alarm rates, were nonetheless able to differentiate targets from noise.

No significant decreases in detection rate were measured over the course of the experiment $\left(\beta_{1}=-0.06\right.$, n.s.). However, there was evidence of changes in performance within a block (Fig. $2 A$ ). Hit rates decreased from an average of $80 \%$ for the first trial in a block to $63 \%$ for the final trial. This slope, reflecting change in performance within a block, was statistically significant $\left(\beta_{1}=\right.$ $\left.-0.46, t_{(10)}=3.07, p<0.05\right)$, whereas the average false alarm rate did not change significantly during a block $\left(\beta_{1}=0.03, t_{(10)}=\right.$ 0.16 , n.s.). A decrease in $d^{\prime}$ over the course of a block was observed $\left(\beta_{1}=-0.032, t_{(10)}=4.13, p<0.01\right)$, along with a concurrent increase in criterion $\left(\beta_{1}=0.015, t_{(10)}=2.51, p<0.05\right)$.

We also analyzed the effect of previous trial type and accuracy on the target detection on subsequent trials. Compared with the overall detection rate of $70 \%$, subjects were reliably more likely to detect a target when the target was detected on the previous target-plus-mask trial (not necessarily the immediately preceding trial because a target-only or mask-only trial could have intervened) (Fig. $2 B ; M=77 \%, t_{(10)}=3.28, p<0.01$ ), and were less likely to detect a target when the previous target was also undetected $\left(M=53 \%, t_{(10)}=3.91, p<0.01\right)$. The effect of the immediately preceding trial type (Target-Only, Mask-Only, TargetPlus-Mask) on subsequent detection was then considered. A repeated-measures ANOVA on the current trial detection rate as a function of the preceding trial type was not significant $\left(F_{(2,10)}=\right.$ 0.344 , n.s.).
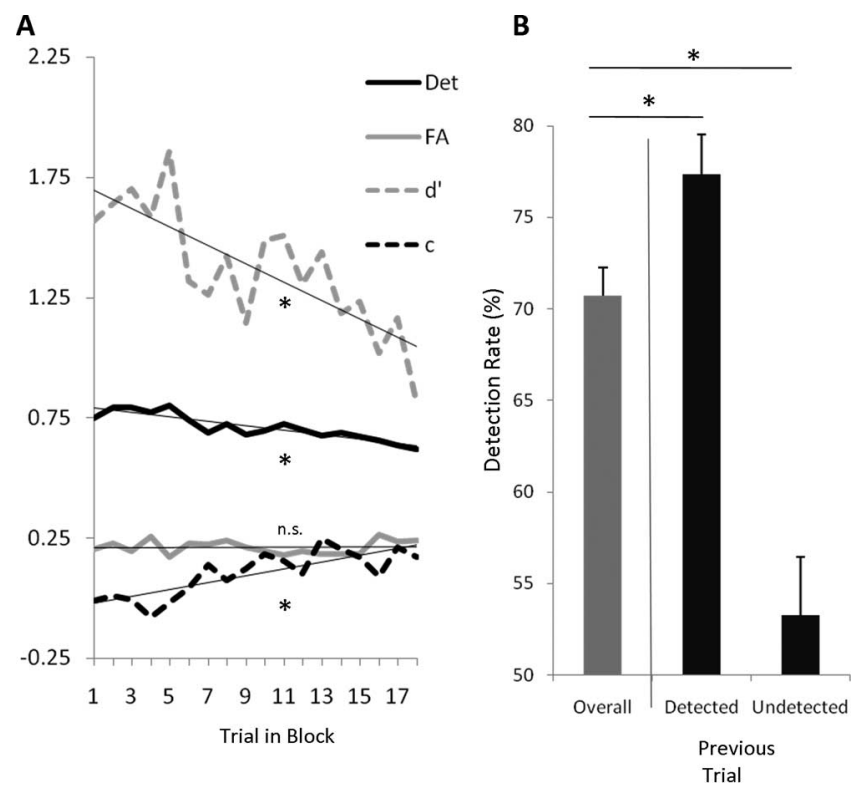

Figure 2. A, Average linear trends over the course of an experimental block for detection rate and false alarm rate (solid lines), and for $d^{\prime}$ and criterion ( $c$; dashed lines). The least squares regression line of best fit for each curve is shown in gray. $\boldsymbol{B}$, Overall detection rate (left) contrasted with detection rate as a function of detection on the previous target-plus-mask trial (center: detected; right: undetected). ${ }^{*} p<0.05$. n.s., Not significant at $\alpha=0.05$; error bars represent within-subject SE.

\section{Electrophysiology}

Table 1 shows the time-window, analyzed electrode location, and mean voltages for detected and undetected targets for each of the ERP components investigated for the fixation and target intervals. Figure 3 depicts the fixation-locked ERPs for detected and undetected target-plus-mask trials. Both the P1 component $\left(t_{(10)}\right.$ $=2.76, p<0.025$, one-tailed) and the $\mathrm{P} 2$ component $\left(t_{(10)}=\right.$ $2.48, p<0.05$, one-tailed) were reliably larger on trials in which the target would later be detected than when it was subsequently undetected. There was no reliable difference in the mean amplitude of the N1 component elicited by fixation for detected compared with undetected targets $\left(t_{(10)}=1.37\right.$, n.s., one-tailed).

We next assessed the effect of $\alpha$ power and phase on target detection. Figure $4 \mathrm{~A}$ shows the decrease in detection performance as a function of increased $\alpha$ power, with the linear trend being significant $\left(F_{(1,10)}=16.43, p<0.005\right)$. This finding was corroborated by a direct comparison of the $\alpha$ power in the period preceding target onset for detected and undetected targets. There was significantly more $\alpha$ power for undetected targets $(M=2.74)$ than for detected targets $\left(M=2.63 ; t_{(10)}=2.67, p<0.025\right)$. In addition, there was a numerical increase in $\alpha$ power from the first ( $M=2.64)$ to the second $(M=2.74)$ half of the block, consistent with the observed decrease in performance over the block, but this effect did not achieve significance $\left(t_{(10)}=1.94, p<0.10\right)$.

Figure $4 B$ shows the difference in detection rate as a function of the phase of $\alpha$ activity at target onset, for both high- and low- $\alpha$ power trials. There were significant main effects of power $\left(F_{(1,10)}\right.$ $=9.15, p<0.05)$ and phase of $\alpha\left(F_{(1,10)}=6.48, p<0.05\right)$. Furthermore, to examine simple main effects, we ran paired twotailed $t$ tests comparing the detection rate for each phase bin, separately for high- and low- $\alpha$ power. They showed, as predicted, that the effect of phase was only reliable for high- $\alpha$ power trials. For low- $\alpha$ power trials, there was no effect of $\alpha$ phase $\left(45-225^{\circ}=\right.$ $0.73,225-45^{\circ}=0.73, t_{(10)}=0.35$, n.s.), whereas at high levels of $\alpha$ power, there was a significant effect of phase $(45-225=0.67$, 
Table 1. ERP component statistics

\begin{tabular}{|c|c|c|c|c|c|}
\hline Event & Component & Electrode & Window (ms) & Det. $(\mu \mathrm{V})$ & Undet. $(\mu \mathrm{V})$ \\
\hline \multirow[t]{3}{*}{ Fixation } & $\mathrm{P} 1$ & $\mathrm{~T} 5$ & $90-160$ & 0.02 & $-0.10^{*}$ \\
\hline & N1 & $\mathrm{Fz}$ & $150-230$ & -0.06 & $-0.16^{*}$ \\
\hline & P2 & $\mathrm{T} 5$ & $200-250$ & 0.34 & $0.18^{*}$ \\
\hline \multirow[t]{4}{*}{ Target } & $\mathrm{P} 1$ & $0 z$ & $90-160$ & 0.23 & $0.08^{* *}$ \\
\hline & N1 & $\mathrm{Fz}$ & $150-230$ & 0.30 & $0.14^{*}$ \\
\hline & $\mathrm{P} 2$ & $0 z$ & $200-250$ & 0.26 & $0.14^{*}$ \\
\hline & P3 & $\mathrm{Pz}$ & $300-500$ & 0.11 & $-0.12^{*}$ \\
\hline
\end{tabular}

${ }^{*} p<0.05 ;{ }^{* *} p<0.01$. Window (ms): time window over which the mean amplitude was taken, with respect to the onset of the time-locking event. Det. ( $\left.\mu \mathrm{V}\right)$ and Undet. $(\mu \mathrm{V})$ : mean amplitude of the ERP over the time window for the detected and undetected trials, respectively.

$\left.225-45^{\circ}=0.71, t_{(10)}=4.53, p<0.005\right)$. Finally, the average $\alpha$ differences (combining phase and amplitude using the Cartesian coordinates of the complex space) were directly compared between detected and undetected trials using a Hotelling's bivariate $F$ test. There was a significant difference in $\alpha$ between detected (circular grand mean $\left.(\mathrm{CGM})=273.5^{\circ}\right)$ and undetected target conditions $\left(\mathrm{CGM}=96.4^{\circ}\right.$; $\left.F_{(2,9)}=22.00, p<0.001\right)$. Although this effect may have also been influenced by differences in $\alpha$ power, Figure $4 C$ and the above-mentioned analysis presented in $4 B$ clearly show an effect of phase. Figure $4 C$ shows a circular histogram of the difference between each subject's average phase for detected and undetected trials, illustrating that the difference in phase fell between 225 and $315^{\circ}$ for the majority of subjects.

Figure $4 D$ shows two overlaid circular histograms of each subject's mean phase for detected and undetected targets. A nonparametric Monte-Carlo test compared the distribution of mean phases between the detected and undetected conditions. To test the hypothesis that the undetected targets had a reliably different distribution of phase of $\alpha$ at their onset than the detected targets, the observed frequency of mean phases before detected targets falling in each of four bins $\left(45-115^{\circ}, 115-225^{\circ}, 225-315^{\circ}, 315-45^{\circ}\right)$ was computed and used to create a polynomial distribution from which 10,000 random samples of 'observed' distributions were created. An uncorrected $\chi^{2}$ statistic comparing the true observed distribution of mean $\alpha$ phase for undetected targets to the mean phase distribution for detected targets $\left(\chi^{2}=7.58\right)$ was $>9568$ of the $\chi^{2}$ statistics calculated from the randomly generated detected phase distribution $\left(p\left(\mathrm{H}_{0}\right)<0.05\right)$.

To ensure that the greater number of trials present in the detected target condition was not affecting the measures of phase, a bootstrap procedure (Efron, 1979) was used, matching the number of trials in the two conditions. For each subject, trials were selected randomly with replacement from the detected trials, such that they matched the number of undetected trials for that subject (except for one subject who had more undetected trials, in which the processes was reversed). Each subject's mean phase in each condition was then computed, and the grand average over subjects was determined. Figure $4 E$ shows the results of this process being iterated 10,000 times, creating a distribution of sample means for each condition. A clear separation between the mean phases for detected and undetected targets is apparent even when equal numbers of trials between the conditions are used. To quantify the results of this analysis, we measured, for each iteration, whether the mean phase for undetected targets had a greater $\mathrm{X}$ component than the mean phase for detected targets. Of the 10,000 samples, 9787 showed this pattern $\left(p\left(\mathrm{H}_{0}\right)<0.05\right)$. Additionally, the detection rate for the two opposite phase bins was computed for each iteration. On 9835 of the iterations, the detection rate for $225-45^{\circ}$ was greater than for $45-225\left(p\left(\mathrm{H}_{0}\right)<0.05\right)$. Thus, these phase differences are reliable and cannot be attributed to the uneven number of trials in each condition or to amplitude differences that might have influenced the Hotelling test.

It should be noted that although the phase of $\alpha$ was predictive of target detection (Fig. $4 F$ ), there was on average no phase-locking to the expected onset of the target from the onset of the fixation cross. This was demonstrated in the following way. For each subject, an average was computed across all trials (disregarding whether they were trials in which the target was detected or undetected). The resulting grand average is shown as a gray line in Figure $4 F$. As can be seen from this figure, this average produces a flat line during the pretarget period, demonstrating that, on average, the phase of $\alpha$ in the period immediately preceding the target appearance was random. This indicates that the phase of $\alpha$ per se is unrelated to the presentation of the fixation stimulus, and therefore its relationship 
A

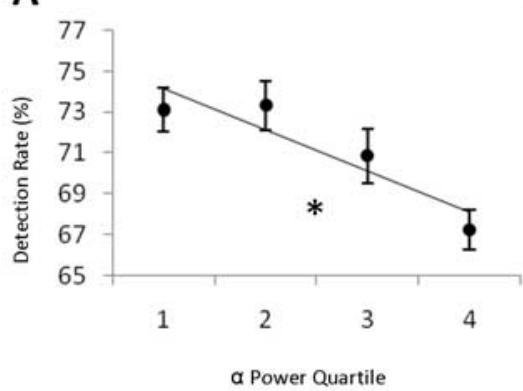

C

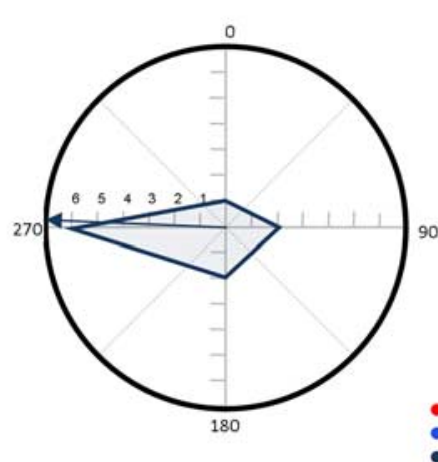

$\mathbf{E}$

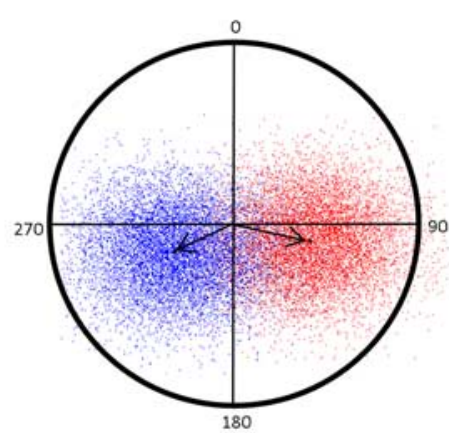

B

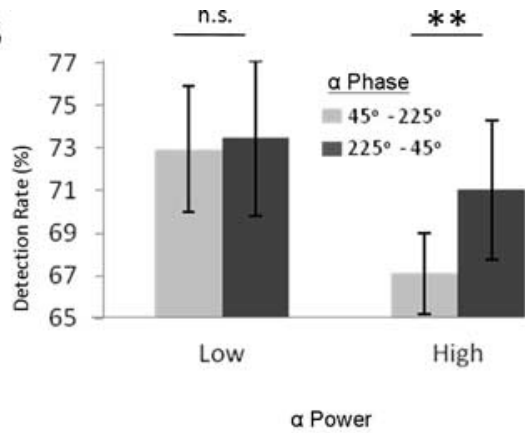

D

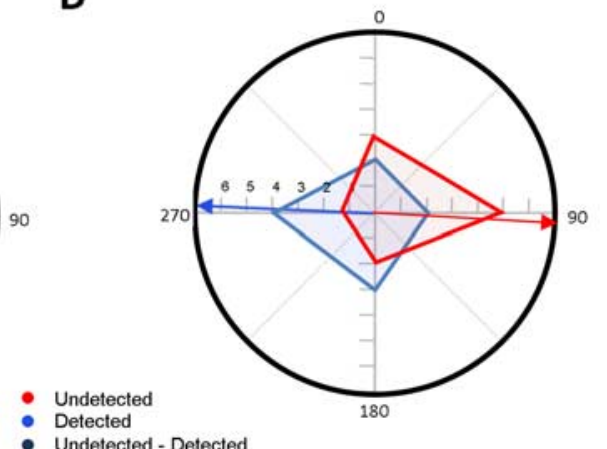

Undetected - Detected

$\mathbf{F}$

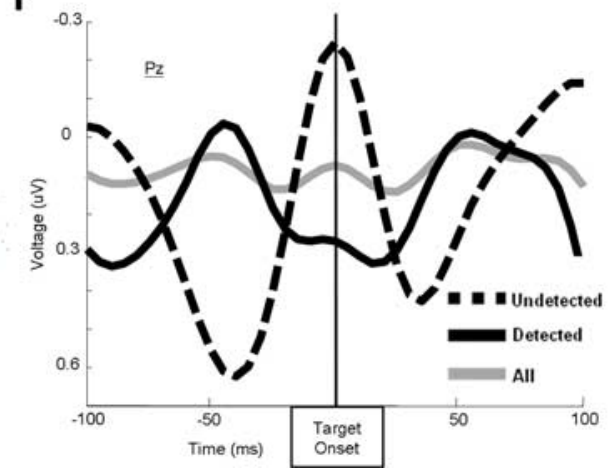

Figure 4. A, Detection rate plotted as a function of prestimulus log-transformed $\alpha$ power quartiles revealing a significant decrease in target detection with increased power; ${ }^{*} p<0.05$. $\boldsymbol{B}$. Detection rate plotted as a function of opposite phases of prestimulus $\alpha$ phase, comparing high- and low-power trials. The effect of phase was only present for high power trials. ${ }^{* *} p<$ 0.005 ; n.s., not significant at $\alpha=0.05$; error bars represent within-subject SE. C, Circular histogram of the difference in mean phase of $\alpha$ activity between detected and undetected targets indicating a non-zero difference in phase. Distance from origin reflects the number of subjects whose difference vector falls within each phase quadrant, indicated by dashed gray lines. The blue arrow represents the average difference in mean phase across subjects. $\boldsymbol{D}$, Two overlaid circular histograms of the mean phase of $\alpha$ activity preceding both detected (blue) and undetected (red) targets. Arrows represent the average phase of $\alpha$ across subjects for each condition. $\boldsymbol{E}$, Results of a bootstrap analysis of the mean phase of $\alpha$ activity preceding detected and undetected targets. An equal number of trials were randomly sampled from each condition with replacement for each subject. The grand average phase of each of these samples was obtained for each condition and plotted on the chart. Vectors represent the mean across all bootstrapped samples. There is a clear separation of the mean phase of $\alpha$ between the two conditions, equating trial numbers. F, Grand average ERP at channel Pz in the $100 \mathrm{~ms}$ preceding and after the onset of the target. Note the opposite phase for the undetected (dashed) and detected (solid) targets. When collapsed across all targets, no phase-locking is apparent (gray).

with detection accuracy is not a spurious correlation between $\alpha$ phase at fixation onset and detection accuracy.

Figure 5 shows the ERPs for detected and undetected targets, which were obtained by subtracting the mask-only condition from the corresponding target-plus-mask condition. The mean amplitudes of the P1 $\left(t_{(10)}=3.10, p<0.01\right.$, one tailed $)$, N1 $\left(t_{(10)}\right.$ $=2.66, p<0.025$, one-tailed $), \mathrm{P} 2\left(t_{(10)}=2.24, p<0.05\right.$, onetailed), and P3 $\left(t_{(10)}=2.45, p<0.05\right.$, one-tailed) were all reliably greater for detected compared with undetected masked targets. There were no differences between the mean voltage in the baseline period for detected $(M=0.32 \mu \mathrm{V})$ compared with undetected $(M=0.10 \mu \mathrm{V}$; $t_{(10)}=1.13$, n.s.) targets.

Finally, to test the direct effects of the phase of $\alpha$ on the ERPs elicited by targets, trials were resorted based on the phase of $\alpha$ at the onset of the stimulus. The peak amplitude and latency of the N1 component were then measured in the window between 50 and $170 \mathrm{~ms}$ after target onset (to replicate Barry et al., 2004). When the phase of $\alpha$ was between 45 and $225^{\circ}$ (and detection was worse), the N1 component was reliably smaller in peak amplitude $\left(M=-0.14 \mu \mathrm{V}\right.$ vs $-1.42 \mu \mathrm{V}, t_{(10)}=3.89$, $p<0.005)$ and had a longer latency $(M=$ $100 \mathrm{~ms}$ vs $66 \mathrm{~ms}, t_{(10)}=7.21, p<0.0001$ ) than when the phase of $\alpha$ was between 225 and $45^{\circ} . \alpha$ phase did not systematically affect any of the other ERP components.

\section{Discussion}

We assessed the relationship between prestimulus electrophysiological activity and subsequent target detection in a metacontrast masking paradigm. Behavioral results indicated that targets were successfully masked on a moderate proportion of trials, in line with past evidence of similar metacontrast masking effects using comparable stimuli at analogous SOAs (Boyer and Ro, 2007). Importantly, the moderate detection rates allowed us to assess patterns in behavior and differences in neural activity for detected compared with undetected targets under identical stimulus conditions.

The observed decreases in detectability and $\alpha$ power across the course of a block (as manifest in both decreased detection rate and $d^{\prime}$ ) suggest that fluctuations in behavioral and corresponding neural states play a role in visual awareness. Our data indicate systematic increases in target detection when the previous target was detected and corresponding decreases when the previous target was undetected. These results suggest that subjects' performance fluctuates over time, presumably reflecting variations in brain states related to priming, vigilance, preparation, or levels of fatigue, and that by knowing these states at a given point of time, one can predict visual performance and awareness of subsequent events.

We also observed $\mathrm{P} 1$ and $\mathrm{P} 2$ amplitude increases in response to the fixation cross for trials on which the target was later detected, suggesting that specific state changes occurring before the target appears can influence its detection. These phenomena resemble the upregulation of these visual ERP components by attention (Luck et al., 2000) and are consistent with a brain state 


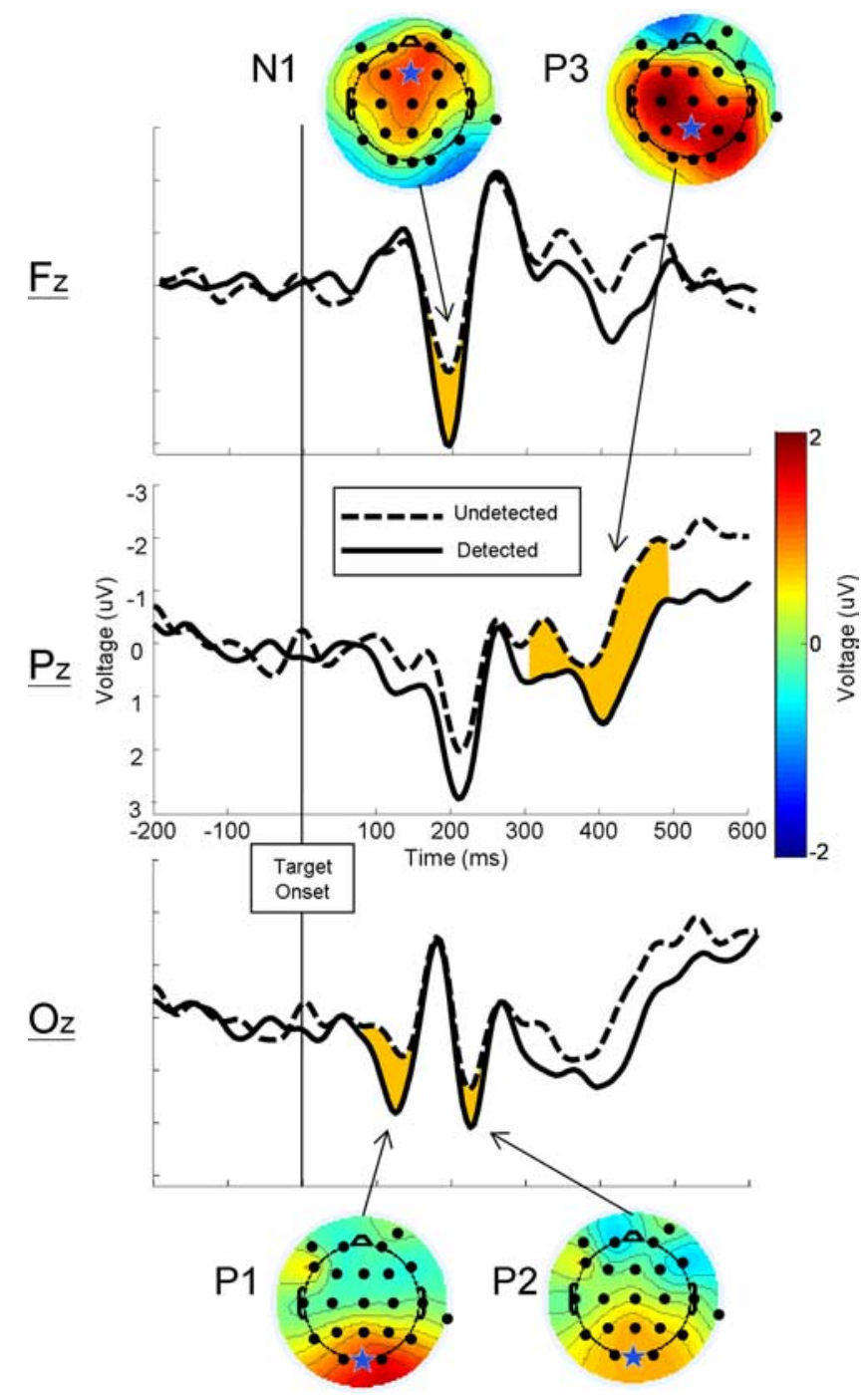

Figure 5. Difference waves created by subtracting ERPs on mask-only trials from masked target trials, yielding waveforms representing the activity resulting from the detected (solid) and undetected (dashed) targets, plotted at three locations ( $\mathrm{Fz}, \mathrm{Pz}, \mathrm{Oz})$ along the midline. Shaded waveform regions represent analyzed time windows in which the mean voltage was reliably greater for detected targets than for undetected targets. Stars represent channels from which analysis measurements were taken for each component. Interpolated scalp voltage maps of the difference between detected and undetected trials at the peak difference for components P1, N1, P2, and P3.

geared for enhanced processing that is maintained until target onset. Similarly, Pourtois et al. (2006), using a task in which subjects must detect changes between pairs of images, reported that the ERPs evoked by the first stimulus of the pair (i.e., before the change) were larger for trials on which the change would be subsequently detected. These results make intuitive sense in that enhanced processing of the first stimulus should lead to an improved comparison with the second. However, in our case, there is no reason to expect that increased processing of the fixation cross itself, which is not predictive of target presence, should lead to enhanced performance. In fact, one might expect that increased processing at fixation would lead to reduced target visibility attributable to forward masking. Instead, we show that target processing is enhanced when previous fixation processing is upregulated, suggesting that the same enhanced brain state influences both fixation processing and target detection.

The possibility that brain state plays an important role in tar- get detection is further corroborated by the analyses of $\alpha$ power and phase in the period preceding target onset. Specifically, we found decreased detection as a function of increased power, in line with past evidence (Ergenoglu et al., 2004; Thut et al., 2006; Hanslmayr et al., 2007; van Dijk et al., 2008; but see LinkenkaerHansen et al., 2004; Babiloni et al., 2006). We also found increases in $\alpha$ power over the course of a block, concomitant with decreases in detection.

Crucially, we show for the first time that the phase of $\alpha$ systematically influences whether a visual stimulus will reach awareness. Detection rate reliably differed between opposite phases of $\alpha$, but only for trials with high $\alpha$ power. This finding provides evidence that oscillations of $\alpha$ activity represent oscillations in cortical excitability (Lindsley, 1952).

\section{A “pulsed inhibition” account of $\alpha$}

Our results demonstrate that both $\alpha$ power and phase differences contribute to conscious target detection. $\alpha$ power suppression has been proposed to be a manifestation of controlled enhancements in attention (Worden et al., 2000; Fries et al., 2001; Sauseng et al., 2005). However, the oscillatory nature of $\alpha$ suggests that attention level is not static but oscillates between cortical excitability and inhibition at a frequency of $\sim 10$ times/s (which we label "microstate"). In fact, our data suggest that inhibition is not equal across the $\alpha$ cycle, but only manifests its effects on behavior during part of the cycle, generating a form of "pulsed inhibition." This "pulsed inhibition" would only occur when $\alpha$ is present, leading to oscillations of cortical inhibition which, averaged over time, appear as more static inhibitory states. In contrast, the controlled or spontaneous suppression of $\alpha$ would diminish these periods of cortical inhibition, leading to more prolonged periods of excitation. In other words, we are proposing two routes to detection: 1) during controlled suppression of $\alpha$, and 2) during the excitatory phase of $\alpha$, when $\alpha$ is not suppressed. In this "pulsed inhibition" framework (which we label the " $\alpha$ braking system," or ABS, for analogy to the servo-mechanisms controlling car breaks), the power of $\alpha$ activity is controlled, whereas the pulsating inhibition it entails operates largely on its own. Similar to a car's anti-lock brake system, this mechanism would allow contact with the environment even when inhibition is engaged.

This proposed oscillatory mechanism by which $\alpha$ exerts its inhibitory influence is compatible with the inhibition-timing hypothesis of $\alpha$ (Klimesh et al., 2007), in which $\alpha$ represents a mechanism of top-down inhibitory and temporal control. However, our account emphasizes the oscillatory nature of $\alpha$ in the attentional domain as useful for continued yet reduced contact with the inhibited modality.

A complementary role of $\alpha$ oscillations as representing perceptual packets of information has been recently summarized by Van Rullen and Koch (2003). Among the evidence for this account is a study by Gho and Varela (1988), indicating that if two flashes of light are presented in close temporal succession, at one particular $\alpha$ phase they are interpreted as occurring simultaneously, whereas in the opposite phase they are reported as occurring in succession. Our results could be interpreted under this account in that at a certain $\alpha$ phase the target and mask would be fused, diminishing target detectability.

Recently, Lakatos et al. (2008) showed that the phase of $\delta$-band oscillations can be controlled to increase cortical excitability at attended stimulus onset. However, our data do not support the proposal that the phase of $\alpha$ can be similarly controlled because, across all trials, the phase of $\alpha$ was random, even though the interval between the onset of fixation and that of the 
target was constant (Fig. $4 F$ ). Instead, these data support the notion of a dual route to detection: a more controlled route, via $\alpha$ suppression, and a less controlled route, determined by the timing of the stimulus relative to the "pulsed inhibition."

These findings demonstrate that the phase of $\alpha$ at stimulus onset can also influence subsequent ERPs (also see Trimble and Potts, 1975; Jansen and Brandt, 1991; Haig and Gordon, 1998; Kruglikov and Schiff, 2003; Barry et al., 2003, 2004; Becker et al., 2008). The reduced brain activation manifested by the reduced and delayed N1 amplitude may be the ultimate factor affecting the probability of stimulus detection. Our data reveal that undetected targets (which were below the threshold for awareness) elicited an analogous, albeit smaller, electrophysiological response than did targets for which subjects reported awareness. This result is consistent with the finding by Wyart and TallonBaudry (2008) of a dissociation between anticipatory top-down activity and awareness itself. Modulations of $\alpha$ phase and power, which are associated with cortical excitability, may merely enhance or suppress visual processing. However, visual awareness may only occur when, based in part on the modulatory top-down activity, the brain response exceeds a given activation threshold. In other words, it seems that the qualitative behavioral difference between detected and undetected trials depends on whether the amplitude of the modulated neural response exceeds an internal threshold. Such a model also allows for subthreshold processing of undetected visual events (Ro, 2005).

\section{Reentrant processing}

The observed differences in the P1 time-range, purportedly associated with extrastriate and fusiform areas (Clark and Hillyard, 1996), indicate that neural processes as early as $100 \mathrm{~ms}$ poststimulus reflect differences in visual target detection and awareness, echoing the finding by Pins and Ffytche (2003) that the first correlates of consciousness occur in the $100 \mathrm{~ms}$ time range. In a previous study using single-pulse transcranial magnetic stimulation, it was shown that feedback activity to primary visual cortex within the time frame of the target-to-mask SOA (i.e., $42 \mathrm{~ms}$ ) is important for metacontrast masking effectiveness and visual awareness (Ro et al., 2003). These findings are consistent with the computational model of object substitution (CMOS) (Di Lollo et al., 2000), implicating reentrant connections into early visual areas as important for visual awareness.

\section{Conclusions}

This study reveals the influence of oscillatory microstates of cortical activity, manifested by $\alpha$ phase, on subsequent neural activity and visual awareness. In addition, both $\alpha$ power and larger fixation-locked ERPs are predictive of the detectability of masked visual targets. A "pulsed inhibition" (or ABS) theory of cortical $\alpha$-band activity can account for the observed results. When $\alpha$ power is large its phase can influence some ERP components and reduce detection rates, consistent with a framework in which visual awareness arises when a particular threshold for neural activity is surpassed.

\section{References}

Babiloni C, Vecchio F, Bultrini A, Romani GL, Rossini PM (2006) Pre- and post-stimulus alpha rhythms are related to conscious visual perception: a high-resolution EEG study. Cereb Cortex 16:1690-1700.

Barry RJ, de Pascalis V, Hodder D, Clarke AR, Johnstone SJ (2003) Preferred EEG brain states at stimulus onset in a fixed interstimulus interval auditory oddball task, and their effects on ERP components. Int J Psychophysiol 47:187-198.

Barry RJ, Rushby JA, Johnstone SJ, Clarke AR, Croft RJ, Lawrence CA (2004)
Event-related potentials in the auditory oddball as a function of EEG alpha phase at stimulus onset. Clin Neurophysiol 115:2593-2601.

Becker R, Ritter P, Villringer A (2008) Influence of ongoing alpha rhythm on the visual evoked potential. Neuroimage 39:707-716.

Boyer J, Ro T (2007) Attention attenuates metacontrast masking. Cognition 104:135-149.

Breitmeyer BG, Ogmen H (2000) Recent models and findings in visual backward masking: a comparison, review, and update. Percept Psychophys 62:1572-1595.

Callaway E, Yeager CL (1960) Relationship between reaction time and electroencephalographic alpha phase. Science 132:1765-1766.

Clark VP, Hillyard SA (1996) Spatial selective attention affects early extrastriate but not striate components of the visual evoked potential. J Cogn Neurosci 8:387-402.

Del Cul A, Baillet S, Dehaene S (2007) Brain dynamics underlying the nonlinear threshold for access to consciousness. PLoS Biol 5:e260.

Delorme A, Makeig S (2004) EEGLAB: an open source toolbox for analysis of single-trial EEG dynamics. J Neurosci Methods 134:9-21.

Di Lollo V, Enns JT, Rensick RA (2000) Competition for consciousness among visual events: the psychophysics of reentrant visual processes. J Exp Psychol Gen 129:481-507.

Efron B (1979) Bootsrap methods: another look at the jackknife. Ann Statistics 7:1-26.

Ergenoglu T, Demiralp T, Bayraktaroglu Z, Ergen M, Beydagi H, Uresin Y (2004) Alpha rhythm of the EEG modulates visual detection performance in humans. Cogn Brain Res 20:376-383.

Fahrenfort JJ, Scholte HS, Lamme VAF (2007) Masking disrupts reentrant processing in human visual cortex. J Cogn Neurosci 19:1488-1497.

Fries P, Reynolds JH, Rorie AE, Desimone R (2001) Modulation of oscillatory neuronal synchronization by selective visual attention. Science 291:1560-1563.

Gho M, Varela FJ (1988) A quantitative assessment of the dependency of the visual temporal frame upon the cortical rhythm. J Physiol 83:95-101.

Gratton G, Coles MGH, Donchin E (1983) A new method for off-line removal of ocular artifact. Electroencephalogr Clin Neurophysiol 55:468-484.

Haig AR, Gordon E (1998) EEG alpha phase at stimulus onset significantly affects the amplitude of the P3 ERP component. Int J Neurosci 93:101-116.

Hanslmayr S, Aslan A, Staudigl T, Klimesch W, Herrmann CS, Bauml K (2007) Prestimulus oscillations predict visual perception performance between and within subjects. Neuroimage 37:1465-1473.

Haynes JD, Driver J, Rees G (2005) Visibility reflects dynamic changes of effective connectivity between V1 and fusiform cortex. Neuron 46:811-821.

Jansen BH, Brandt ME (1991) The effect of the phase of prestimulus alpha activity on the average visual evoked response. Electroencephalogr Clin Neurophysiol 80:241-250.

Klimesch W, Sauseng P, Hanslmayr S (2007) EEG alpha oscillations: The inhibition-timing hypothesis. Brain Res Rev 53:63-88.

Kruglikov SY, Schiff SJ (2003) Interplay of the electroencephalogram phase and auditory-evoked neural activity. J Neurosci 23:10122-10127.

Lakatos P, Karmos G, Mehta AD, Ulbert I, Schroeder CE (2008) Entrainment of neuronal oscillations as a mechanism of attentional selection. Science 320:110-113.

Lau HC, Passingham RE (2002) Relative blindsight in normal observers and the neural correlate of visual consciousness. Proc Natl Acad Sci U S A 103:18763-18768.

Lindsley DB (1952) Psychological phenomena and the electroencephalogram. Electroencephalogr Clin Neurophysiol 4:443-456.

Linkenkaer-Hansen K, Nikulin VV, Palva S, Ilmoniemi RJ, Palva JM (2004) Prestimulus oscillations enhance psychophysical performance in humans. J Neurosci 24:10186-10190.

Luck SJ, Woodman GF, Vogel EK (2000) Event-related potential studies of attention. Trend Cogn Sci 4:432-440.

Pins D, Ffytche D (2003) The neural correlates of conscious vision. Cereb Cortex 13:461-474.

Pourtois G, De Pretto M, Hauert C, Vuilleumier P (2006) Time course of brain activity during change blindness and change awareness: performance is predicted by neural events before change onset. J Cogn Neurosci 18:2108-2129. 
Ramachandran VS, Cobb S (1995) Visual attention modulates metacontrast masking. Nature 373:66-68.

Ro T (2005) Neural mechanisms for conscious and unconscious vision. In: The first half second (Breitmeyer B, Ogmen H, eds). Cambridge, MA: MIT.

Ro T, Breitmeyer B, Burton P, Singhal N, Lane D (2003) Feedback contributions to visual awareness in human occipital cortex. Curr Biol 11:1038-1041.

Romei V, Brodbeck V, Michel C, Amedi A, Pascual-Leone A, Thut G (2008) Spontaneous fluctuations in posterior a-band EEG activity reflect variability in excitability of human visual areas. Cereb Cortex 19:2010-2018.

Sauseng P, Klimesch W, Stadler W, Shabus M, Doppelmayr M, Hanslmayr S, Gruber WR, Birbaumer N (2005) A shift of visual spatial attention is selectively associated with human EEG alpha activity. Eur J Neurosci 22:2917-2926.

Shelley-Tremblay J, Mack A (1999) Metacontrast masking and attention. Psychol Sci 10:508-515.

Tata MS (2002) Attend to it now or lose it forever: Selective attention, metacontrast masking, and object substitution. Percept Psychophys 64:1028-1038.

Thut G, Nietzel A, Brandt S, Pascual-Leone A (2006) Alpha-band electroen- cephalographic (EEG) activity over occipital cortex indexes visuospatial attention bias and predicts visual target detection. J Neurosci 26:9494-9502.

Trimble JL, Potts AM (1975) Ongoing occipital rhythms and the VER. I. Stimulation at peaks of the alpha-rhythm. Invest Ophthalmol 14:537-546.

van Aalderen-Smeets SI, Oostenveld R, Schwarzbach J (2006) Investigating neurophysiological correlates of metacontrast masking with magnetoencephalography. Adv Cogn Psychol 2:21-25.

van Dijk H, Schoffelen JM, Oostenveld R, Jensen O (2008) Prestimulus oscillatory activity in the alpha band predicts visual discrimination ability. J Neurosci 28:1816-1823.

Van Rullen R, Koch C (2003) Is perception discrete or continuous? Trends Cogn Sci 7:207-213.

Worden M, Foxe JJ, Wang N, Simpson GV (2000) Anticipatory biasing of visuospatial attention indexed by retinotopcially specific alpha-band electroencephalography increases over occipital cortex. J Neurosci 20:RC63:1-6

Wyart V, Tallon-Baudry C (2008) Neural dissociation between visual awareness and spatial attention. J Neurosci 28:2667-2679. 